\title{
Environment-Induced Surface Dynamics of a Biomimetic Ionomer Studied Using in Situ Second Harmonic Generation
}

\author{
Ken Welch, ${ }^{*}{ }^{\dagger}$ Matthias Burza ${ }^{\dagger}$ Emad Mukhtar, ${ }^{\dagger}$ and Maria Strømme ${ }^{\dagger}$ \\ Department of Engineering Sciences, The Ångström Laboratory, Uppsala University, Box 534, \\ SE-751 21 Uppsala, Sweden, and Department of Photochemistry and Molecular Science, \\ The Ångström Laboratory, Uppsala University, Box 523, SE-751 20 Uppsala, Sweden
}

Received: May 8, 2008; Revised Manuscript Received: June 6, 2008

\begin{abstract}
The environmental-induced surface dynamics of the biomimetic phosphoryl choline (PC)-functionalized poly(trimethylene carbonate) ionomer has been studied and compared to its unfunctionalized counterpart using in situ second harmonic generation measurements. Whereas the nonpolar liquid $n$-hexane did not induce any surface dynamic processes in the ionomer under study, the presence of water initiated a Debye-type dynamic reaction at the surface of the $\mathrm{PC}$ ionomer, which had no equivalent in the unfunctionalized material. This first-order reaction was attributed to a surface enrichment process of the functionalized ionomer in the hydrophilic environment involving movement of the PC endgroups from aggregates in the bulk to the surface. The time constant of the process was found to be about $6 \mathrm{~min}$, and the corresponding activation energy was $0.4 \mathrm{eV}$. The dehydration process of the PC-functionalized ionomer in nitrogen gas atmosphere could be described by two time constants, one slightly below $1 \mathrm{~min}$ and the other one just above $13 \mathrm{~min}$. The results presented in this work show that SHG measurements are well suited for the study of polymer surface restructuring dynamics in response to environmental changes. Such information is very important for the successful design and implementation of biomimetic polymers intended for biomedical applications.
\end{abstract}

\section{Introduction}

With the advent of novel synthetic polymers intended for biomedical applications, the understanding of polymer surface structures and dynamics in the aqueous environment has become very important as it is the surface of the polymer that inevitably comes into contact with body fluids. Molecular moieties at the surface of polymers restructure or rearrange in response to their environment, ${ }^{1,2}$ and thus to be able to design materials with the appropriate functionality, it is critical to understand the surface properties, and in particular the dynamics of the molecules at the surface, of the synthetic materials. A successful functionality of these materials is highly dependent on their ability to be accepted by the body, and therefore a common strategy is to incorporate biomimetic properties in their design. For example, the use of hydrophilic polymers such as poly(ethylene glycol) (PEG) or the incorporation of biomimetic groups such as phosphoryl choline (PC) greatly increases the acceptance of these biomaterials in vivo. ${ }^{3,4} \mathrm{PC}$ is the hydrophilic moiety in the naturally occurring phospholipid phosphatidylcholine present in the cell membrane and is a zwitterionic group, meaning that it contains both a positive and a negative charge.

The task of characterizing the polymer surface in the aqueous environment is fraught with difficulties as the interface of interest is buried and difficult to study on the molecular level with most surface sensitive characterization methods. Contact angle measurements can be used to monitor the macroscopic surface structural changes of polymers in contact with water, ${ }^{2,5}$ but they do not provide information on the molecular level. Most

\footnotetext{
* Corresponding author. Phone: +46 (0)18-471 7944. E-mail: ken.welch@ angstrom.uu.se.

${ }^{\dagger}$ Department of Engineering Sciences, Uppsala University.

\$epartment of Photochemistry and Molecular Science, Uppsala University.
}

intrinsically surface-sensitive methods require a vacuum to operate, making it difficult to study interfaces involving liquids, or cannot probe buried surfaces in situ.

Optical techniques generally do not suffer from these limitations and offer a nondestructive means of probing any interface accessible to light in situ. Although linear optical techniques such as infrared and Raman spectroscopy have the ability to probe buried interfaces, their performance is generally limited by the much larger signal originating from the bulk. ${ }^{6}$ On the other hand, the nonlinear optical techniques of second harmonic generation (SHG) and sum frequency generation (SFG) have emerged over the past two decades as the most successful and versatile techniques for investigating buried interfaces with submonolayer sensitivity. ${ }^{7-10}$ With the SHG technique, a laser beam at frequency $\omega$ interacts with the medium to produce an output at double the frequency, $2 \omega$. SFG differs in that two input laser beams, typically one at a visible frequency $\omega_{\mathrm{VIS}}$ and the other at an infrared frequency $\omega_{\mathrm{IR}}$, overlap in the medium to produce a signal at the sum frequency $\omega_{\mathrm{VIS}}+\omega_{\mathrm{IR}}$. The experimental setup is more complex, but has the advantage of providing spectroscopic information by scanning $\omega_{\text {IR }}$ through the resonant frequencies of the molecular bonds of interest. As second-order optical processes, generation of the SHG/SFG signal is theoretically forbidden in the bulk of a material with inversion symmetry, ${ }^{11}$ such as an amorphous polymer (in the dipole approximation). At the surface, inversion symmetry is necessarily broken, even at the interface between two materials with inversion symmetry, and therefore these techniques are ideal for providing virtually background-free information about buried interfaces in situ with high spatial, temporal, and spectral resolution. Furthermore, information about molecular orientation and conformation at the interface can be ascertained by using different polarization combinations of the input and output beams. ${ }^{12,13}$ 
The polymer/water interface has been studied extensively with SFG during the past decade,,, $14-18$ providing information about ordering, orientation, and conformation of the polymer surface. However, there have been very few studies ${ }^{15}$ of the dynamics of the surface molecular changes in response to alteration of environment. This is perhaps partly due to the fact that a SFG spectrum normally takes several minutes to obtain. On the other hand, SHG can provide high temporal resolution and has often been used in molecular dynamics studies at the liquid/solid interface, such as self-assembled monolayer formation kinetics ${ }^{19}$ or protein adsorption. ${ }^{20,21}$ However, to the best of our knowledge, SHG has not been used to study environment-induced dynamics of polymer surfaces.

In this work, we use SHG to study a recently developed biomimetic ionic polymer (ionomer), consisting of a poly(trimethylene carbonate) (PTMC) polymer backbone functionalized with PC endgroups. This biodegradable ionomer has been shown to exhibit dual activity with the zwitterionic PC group forming physical cross-links in the bulk, as well as providing a hydrophilic and hemocompatible surface. ${ }^{22}$ It is the surface dynamics of the polar PC moieties that are of particular interest as they are expected to respond to the environment in contact with the surface of the ionomer. Earlier attempts using PC endfunctionalized poly( $\varepsilon$-caprolactone), being semicrystalline, demonstrated that lack of molecular mobility suppressed the possibility of spontaneous surface rearrangement. ${ }^{23}$ Conversely, the use of the low glass transition temperature $\left(T_{\mathrm{g}}=-40^{\circ} \mathrm{C}\right)$ PTMC introduced enough mobility for spontaneous surface enrichment of the hydrophilic PC-group against water. In the bulk, most likely the PC endgroups are captured in zwitterionic aggregates, ${ }^{22,24,25}$ where they form physical cross-links that restrict molecular mobility. The formation of aggregates results in enhanced mechanical properties as compared to the unfunctionalized backbone polymer, PTMC. At ambient and physiological temperatures, PTMC behaves as an amorphous melt and has no mechanical integrity, whereas the PC ionomer behaves like a rubber with a shear modulus of several $\mathrm{MPa}^{22}$ The bulk molecular dynamics of these materials have been recently studied using broadband dielectric spectroscopy where the importance of the PC endgroups for the functionality of the ionomer has been observed. ${ }^{25}$

The molecular surface dynamics of the PC ionomer are studied in this work by altering the environment in contact with the ionomer and observing the change in the SHG signal. Both liquid (polar and nonpolar) and gaseous environments are used. The surface dynamics of the unfunctionalized ionomer backbone, PTMC, are then contrasted with those of the PC ionomer to determine the contribution of the PC endgroups. Additionally, the temperature dependence of the $\mathrm{PC}$ ionomer surface dynamics with the introduction of water is investigated over a temperature range of $10-70{ }^{\circ} \mathrm{C}$. The information gained from this study, coupled with previous studies of the effect of the PC endgroups on the bulk properties of the PC ionomer, can be used to gain a better understanding of the effect of the PC endgroups on the functionality of this biomimetic ionomer in vivo.

\section{Experimental Section}

Materials. The low molecular weight polycarbonate PTMC, Figure 1a, was synthesized by ring-opening polymerization of tri(methylene carbonate), initiated from 1,4-butanediol. The degree of polymerization (DP) was 40 ( $n=20$ on each side), as obtained by ${ }^{1} \mathrm{H}$ NMR endgroup analysis, giving a molecular weight of approximately $4000 \mathrm{~g} / \mathrm{mol}^{22}$ The ionomer, Figure $1 \mathrm{~b}$, was subsequently prepared by functionalizing both ends of the
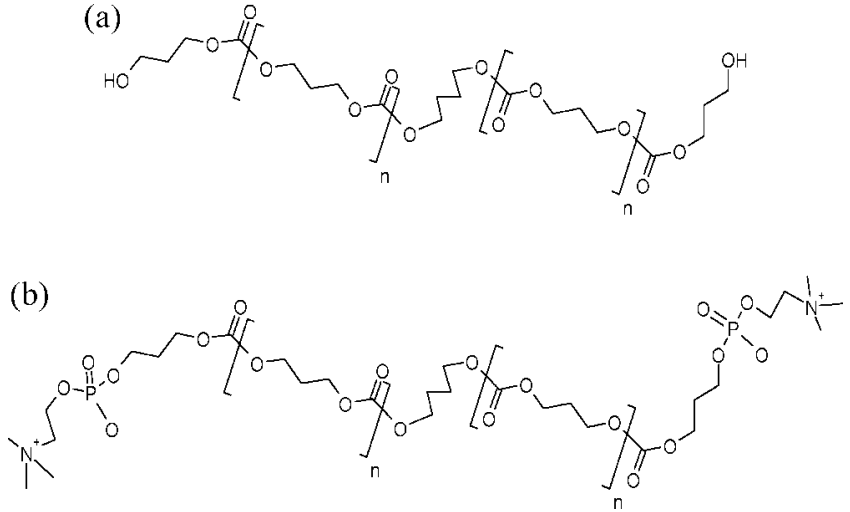

Figure 1. Molecular structure of (a) PTMC $(n \approx 20)$ and (b) PC ionomer $(n \approx 20)$.

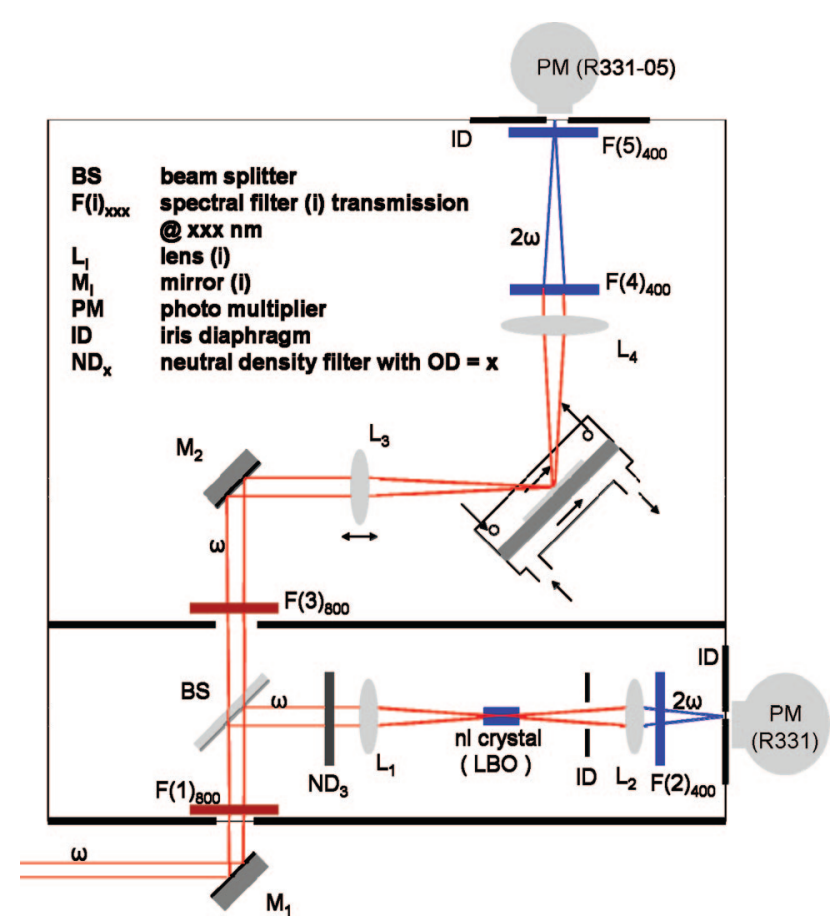

Figure 2. Experimental setup used for SHG measurements.

PTMC polymer with the zwitterionic PC group. Investigation of both PTMC and the ionomer using a spectrophotometer (Agilent 8453) showed no absorption bands in the visible region or at the laser fundamental that might otherwise give rise to resonant enhancements of the SHG process.

Sample Preparation. An $\sim 250 \mu \mathrm{m}$ thick PTMC or ionomer layer was created by casting the polymer from its dichloromethane solution ( $\sim 5 \mathrm{wt} \%$ polymer $)$ onto the glass substrate. Solvent was removed by letting it evaporate for $2 \mathrm{~h}$ under a nitrogen air flow and then placing the sample in a vacuum oven at a temperature of $40{ }^{\circ} \mathrm{C}$ for $\sim 24 \mathrm{~h}$. Such a relatively thick sample ensured that there would be no thickness-dependent effects on the surface dynamics of the polymers.

Experimental Setup. A schematic of the experimental setup used for the SHG measurements is shown in Figure 2. The pulse laser system consists of a combination of a continuous wave pump laser (Verdi-V18, Coherent, USA), a mode-locked Ti: Sapphire laser (Mira 900, Coherent, USA), and a high repetition rate Ti:Sapphire regenerative amplifier (RegA 9000, Coherent, USA), which together produce laser pulses centered around 800 $\mathrm{nm}$ with a pulse width of $240 \mathrm{fs}$ and at a selectable repetition rate of $10-250 \mathrm{kHz}$. A repetition rate of $40 \mathrm{kHz}$ was used in 


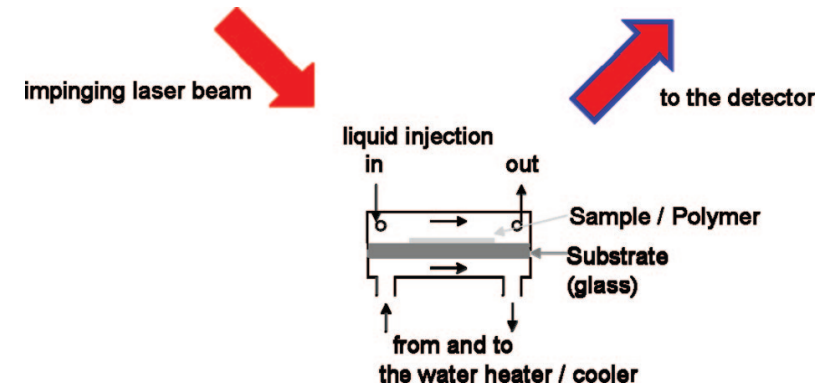

Figure 3. Sample chamber. The environment in contact with the sample can be controlled by injecting the desired liquid or gas. The backside of the glass substrate is in contact with a water flow, which regulates the sample temperature.

this study. The experiment is contained in a box, which is sealed from stray photons with the help of a spectral filter at the entrance hole for the incident laser beam. Inside the box, a beam splitter divides the laser beam into a reference and a sample beam and the frequency doubled photons are detected by photomultiplier tubes (PMT) (R331 and R331-05 for the reference and signal channels, respectively; Hamamatsu Photonics, Japan). The reference beam is focused onto a nonlinear crystal (lithium triborate, $\mathrm{LiB}_{3} \mathrm{O}_{5}$ ) whose frequency-doubled emission serves as a reference to compensate for fluctuations in the laser pulses. The sample beam is focused onto the sample at an incidence angle of $45^{\circ}$, and orthogonal to the incoming beam, scattered photons are collected by a condenser lens, spectrally filtered, and directed through a pinhole to the PMT. The focusing lens for the sample beam is adjusted with the help of a stepper motor to allow for precise focusing of the sample beam.

The spectral detection and filtering system consists of a broadband absorption color glass filter as well as a $400 \mathrm{~nm}$ interference filter. Together with PMTs that have a quantum efficiency of $\sim 30 \%$ at the second harmonic wavelength and less than $0.01 \%$ at the fundamental wavelength, a spectral extinction ratio for the signal of better than $10^{-9}$ is achieved. Because of the low signal intensities of the sample signal, single photon counting was employed to maximize the signal-to-noise ratio. Accordingly, pulses from the PMTs were counted by a dual-channel gated photon counter (SR400, Standford Research Systems, Sunnyvale, CA), which was remotely controlled by a personal computer that also recorded the data. A time window of $5 \mathrm{~s}$ was generally required to collect a sufficient number of photons in the sample channel. This count was divided by the normalized photon count in the reference channel, thereby accounting for any fluctuation in the intensity output of the laser system, which typically varied less than a few percent over a period of several minutes.

The sample chamber shown in Figure 3 provides a temperature-controlled environment in which the polymer surface interactions with different media can be studied in situ. The chamber consists of two fluid chambers separated by a glass substrate upon which the polymer has been cast. The front fluid chamber is approximately $2 \mathrm{~mm}$ thick and allows for the introduction and removal of the desired liquid or gaseous environment that interacts with the polymer surface. The rear fluid chamber provides temperature control by allowing a heated or cooled water flow to come in contact with the backside of the sample substrate glass.

Probing the Surface Dynamics with Second Harmonic Generation in Situ. The material property that is measured by the experiment is a component of the second-order susceptibility tensor, $\chi^{(2)}$, of the molecules at the surface. The generation of a second harmonic signal from the bulk is forbidden for materials with inversion symmetry, assuming that the dipole approximation is justified for the amorphous polymers under study. Therefore, the only contribution to the signal stems from the surface where inversion symmetry is necessarily broken. We are interested in the surface dynamics of the polymer as it undergoes changes with the introduction of different liquids, and therefore we measure the time dependence of $\chi^{(2)}$, which can be derived from the intensity, I, of the SHG signal because:

$$
I(2 \omega) \propto\left|\chi^{(2)}\right|^{2} \cdot I(\omega) \cdot I(\omega)
$$

where $\omega$ is the frequency of the input laser beam. A more detailed theory of the SHG process can be found elsewhere. ${ }^{7,11}$

Because we are measuring a second-order effect, very high electric fields are required for the generation of a detectable amount of second harmonic photons. Therefore, it is important to ensure that we do not damage the polymer. We found that using an average power less than $1 \mathrm{~mW}$ (less than $10^{-7} \mathrm{~J} /$ pulse) prevented damage to the samples. This value is comparable to or less than that used in other SHG or SFG studies involving polymers. ${ }^{18,20,26}$ Additionally, it is important that the sample is not heated by the laser during the experiment as this would affect the molecular dynamics. The lack of absorbance bands at the laser wavelength is a benefit in this respect, and the laser power levels used in this study are less than levels that have been verified to prevent heating of thin polymer samples. ${ }^{27}$

Another important consideration in this experiment is the change in focal point of the laser beam due to the different refractive indices of the fluids that are introduced into the sample chamber. To accurately locate the surface of the polymer, a stepping motor that controls the position of the focusing lens was used to scan the focal point through the polymer surface. By scanning through the polymer surface, we were also able to confirm that the signal did indeed arise from the polymer/liquid interface, and not from the bulk or the polymer/substrate interface.

The surface dynamics of the ionomer after the introduction of a polar liquid was investigated by introducing deionized water into the sample chamber and recording the second harmonic intensity (i.e., the photon counting rate) for a period of $\sim 30$ $\min$. The experiment was repeated at several different temperatures in the range $10-70{ }^{\circ} \mathrm{C}$ to determine the temperature dependence of the molecular surface dynamics. The reversibility of the process was also investigated by withdrawing the water from the chamber after $15 \mathrm{~min}$ and introducing a dry nitrogen flow to help speed the evaporation of any remaining water on the surface of the ionomer. The effect of a nonpolar liquid was also investigated by introducing $n$-hexane into the sample chamber containing the ionomer. Finally, a comparison of the surface dynamics of the ionomer to the unfunctionalized PTMC was made by repeating the experiment with deionized water using PTMC as the test polymer.

\section{Results and Discussion}

Identifying the Polymer/Water Interface. As mentioned in the previous section, moving the focal point of the incident laser beam through the thickness of the sample was used to identify the interface of interest. Figure 4 shows the change in the SHG signal as the focal point was scanned from behind the PC ionomer/substrate interface to in front of the $\mathrm{PC}$ ionomer/water interface. The lens was moved in $23 \mu \mathrm{m}$ steps at a rate of 0.2 steps/s. The measured intensity profile shows two overlapping peaks that represent the front and back surface of the ionomer. 


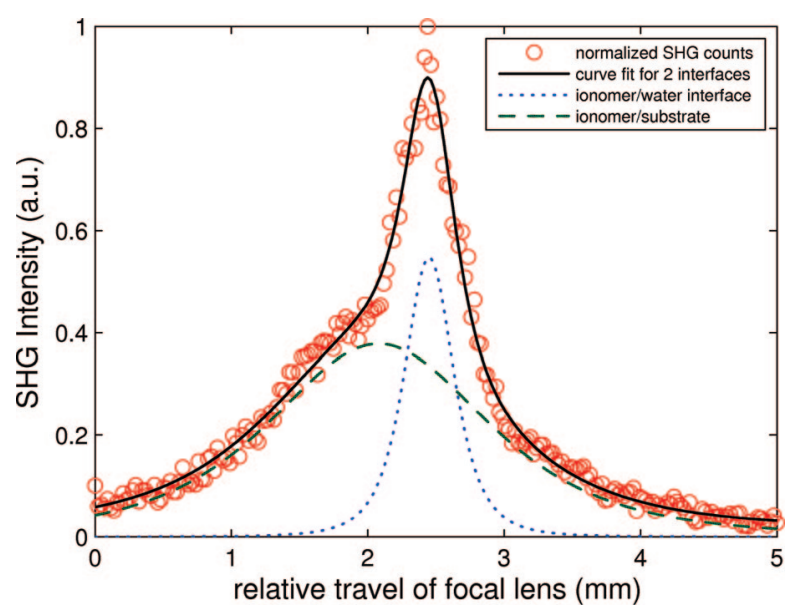

Figure 4. SHG intensity as a function of relative travel of the focal lens with respect to the sample (red $\bigcirc$ ). Two peaks are identified that represent the ionomer/substrate (green dashed curve) and the ionomer/ water (blue dotted curve) interfaces. The solid black line represents a superposition of the above-mentioned two peaks.

The peaks arise when the beam waist of the focused laser beam is centered on the interfaces, where the laser intensity is the greatest. If we know the shape of the beam waist, we can predict the shape of the SHG peaks in Figure 4 because the SHG signal $I_{\mathrm{SHG}}$ is proportional to the square of the incident laser intensity $I$, which in turn is a function of the radius, $r$, of the beam at a distance $z$ from the beam waist:

$$
I_{\mathrm{SHG}}(z) \propto I^{2}(z)=\left[\frac{P}{\pi r^{2}(z)}\right]^{2}
$$

where $P$ is the total power transmitted by the beam. The radius of a focused laser beam at a distance $z$ from the beam waist, assuming a Gaussian beam intensity profile, is given by: ${ }^{28}$

$$
r(z)=r_{\text {focus }} \sqrt{1+\left(\frac{\lambda z}{\pi r_{\text {focus }}^{2}}\right)^{2}}
$$

where $\lambda$ is the wavelength of the laser fundamental and $r_{\text {focus }}$ is the radius of the beam waist. Equation 3 can then be inserted into eq 2 to provide an expression for the SHG signal as a function of distance $z$ from the beam waist. Because this expression applies for normal incidence, the area $\pi r^{2}$ in eq 2 was multiplied by a factor of $\sqrt{ } 2$ as a first-order approximation for the $45^{\circ}$ angle of incidence, and the resulting curve was fitted to the data in Figure 4, assuming two peaks and a small, constant background signal. The distance between the two peaks thus corresponds to the sample thickness, multiplied by $\sqrt{ } 2$ due to the $45^{\circ}$ angle of incidence, and was found to be $260 \mu \mathrm{m} \pm 20 \mu \mathrm{m}$.

From Figure 4, we also observe that the width of the signal peak arising from the polymer/substrate interface is significantly larger than that of the polymer/water interface. Although eqs 2 and 3 suggest that both interfaces should have similar widths, the difference can likely be attributed to the relatively rough upper surface of the cast polymer sample. This imperfect optical interface would distort the wavefront that is focused on the back surface of the polymer, having a significant impact on the generation of the second harmonic signal. Further, deviations to the assumed Gaussian beam intensity profile of the focused laser beam and the fact that the collecting lens L4 (see Figure 2) and PMT aperture were not compensated during the movement of the focusing lens L3 may also lead to different peak shapes than that given by eq 3 . However, the location of the

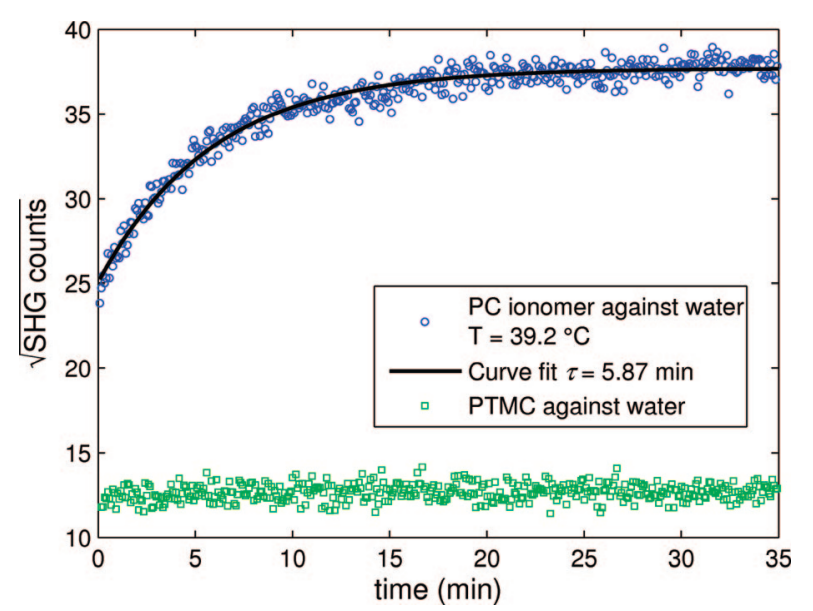

Figure 5. Typical dynamic response curves of $\mathrm{PC}$ ionomer (blue $\mathrm{O}$ ) and PTMC (green $\square$ ) to the introduction of water, taken at 39.2 and $24.4^{\circ} \mathrm{C}$, respectively. The PC ionomer showed first-order kinetics and a time constant $\tau=5.87 \mathrm{~min}$ (black solid line).

peak should be consistent with the locations of the polymer/ water and polymer/substrate interfaces, thus giving a reasonably accurate estimation of the sample thickness as calculated above.

Establishing a Reference: PTMC against Water and PC Ionomer against $\boldsymbol{n}$-Hexane. PTMC serves as an ideal reference sample as it is the polymer backbone of the PC ionomer. By comparing the response of the PTMC surface to the introduction of water with the response of the ionomer with the introduction of water, we can determine the contribution to the molecular dynamics stemming from the polar PC endgroups of the ionomer. Figure 5 displays the SHG signal as a function of time after water is introduced to the surface of PTMC at a temperature of $24.4{ }^{\circ} \mathrm{C}$, as well as to the surface of the ionomer at a temperature of $39.2^{\circ} \mathrm{C}$. The SHG counting rate corresponds to the second harmonic intensity, and therefore the square root of the SHG counting rate is plotted as it is the second-order susceptibility, $\chi^{(2)}$, of the surface that is of interest, as detailed in eq 1. It can be seen from Figure 5 that PTMC exhibited no surface dynamics subsequent to the introduction of water to the surface. This corresponds well with previous contact angle experiments of PTMC where no change in the contact angle of the surface against water was observed after several minutes. ${ }^{29}$ It can be pointed out that the surface dynamics of the PC ionomer are displayed at $39.2^{\circ} \mathrm{C}$ to show the material response at a temperature close to physiological conditions. The PC ionomer also exhibited a similar increase in SHG counts (i.e., an increase in the square root of the SHG counts of 50\%) at room temperature, albeit at a slower rate.

An additional reference measurement was performed by subjecting the surface of the PC ionomer to the nonpolar liquid, $n$-hexane. It was found that the SHG signal did not significantly change over a period of $30 \mathrm{~min}$ when $n$-hexane was introduced to the surface of the ionomer, which had previously been in contact with air. This was expected as both air and nonpolar liquid environments should induce a hydrophobic surface conformation in the ionomer. In fact, previous X-ray photoelectron spectroscopy (XPS) measurements have shown that the polar endgroups of the PC ionomer are not present at the surface of dried ionomer samples. ${ }^{29}$

Surface Dynamics of PC Ionomer. A typical SHG signal resulting from the introduction of water to the surface of the $\mathrm{PC}$ ionomer is displayed in Figure 5. Because we have seen that the backbone of the ionomer (PTMC) shows essentially no response to the introduction of water, we can attribute the 


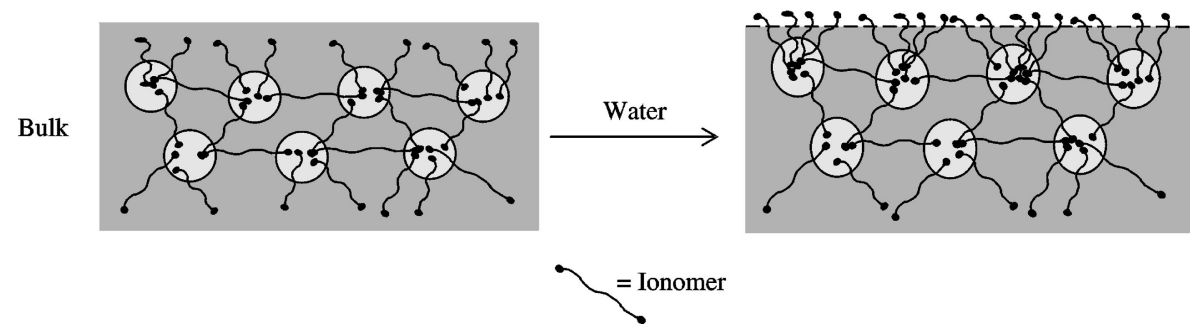

Figure 6. Surface enrichment behavior of the PC ionomer with an added hydrophilic environment.

dynamic nature of the ionomer response to the presence of the PC endgroups. It should be noted that we assume that the SHG signal stems primarily from the interface in contact with water; however, from Figure 4 we know that there may be background contributions from other sources, such as the interface between the ionomer and the substrate. However, these background signals remain constant during the experiment and do not affect the dynamics observed from the ionomer/water interface.

An important consideration when addressing the source of the observed SHG signal is the possible presence of absorbed water in the bulk of the sample. Water uptake could result in swelling of the polymer film, and the ensuing change in interface position with respect to the focal point of the laser may thus produce a change in the SHG signal. Although this possibility cannot be totally excluded because the PC ionomer is known to absorb significant amounts of water, the time scale of the absorption is on the order of $24 \mathrm{~h}^{24}$ and thus should not significantly affect the present measurements.

After the introduction of water to the sample, we expect to measure a larger SHG signal with the ionomer than with PTMC due to the presence and increased ordering of the PC endgroups, as polar groups will generally have larger second-order susceptibilities. ${ }^{30}$ However, at the start of a measurement with the ionomer, one might anticipate SHG signal levels similar to those found with PTMC due to the surface depletion of PC endgroups, as proven by XPS measurements that showed a lack of phosphorus signal at the outermost surface of the dried ionomer. ${ }^{29}$ Comparing the PTMC and PC ionomer responses in Figure 5, we find significantly higher SHG signals with the ionomer at the start of the measurement. This suggests that the presence of the PC endgroups in the ionomer results in a more polar surface as compared to PTMC, even in the dry state. This is supported by advancing contact angle measurements of cast films of both PTMC and the ionomer that show that the dry ionomer surface is more hydrophilic than the dry PTMC surface. ${ }^{29}$

The molecular dynamics of the PC ionomer with the introduction of water suggest first-order kinetics, as shown by the curve fit in Figure 5 corresponding to the equation:

$$
\chi^{(2)}=\sqrt{\text { SHG counts }}=a\left(1-\exp \left(\frac{-t}{\tau}\right)\right)+b
$$

where $a$ and $b$ are constants, $t$ is time, and $\tau$ is the time constant. Simple exponential behavior, like the one seen in Figure 5 and corresponding to a Debye-type relaxation, ${ }^{31}$ is not normally observed with polymer dynamics. ${ }^{32}$ Because of the cooperative interactions between the moieties in the polymer bulk, modified exponential behavior is more common, and literature data are usually more closely fit by models such as the KohlrauschWilliams-Watts $(\mathrm{KWW})$ stretched exponential. ${ }^{33}$ Figure 6 shows a schematic representation of the postulated surface

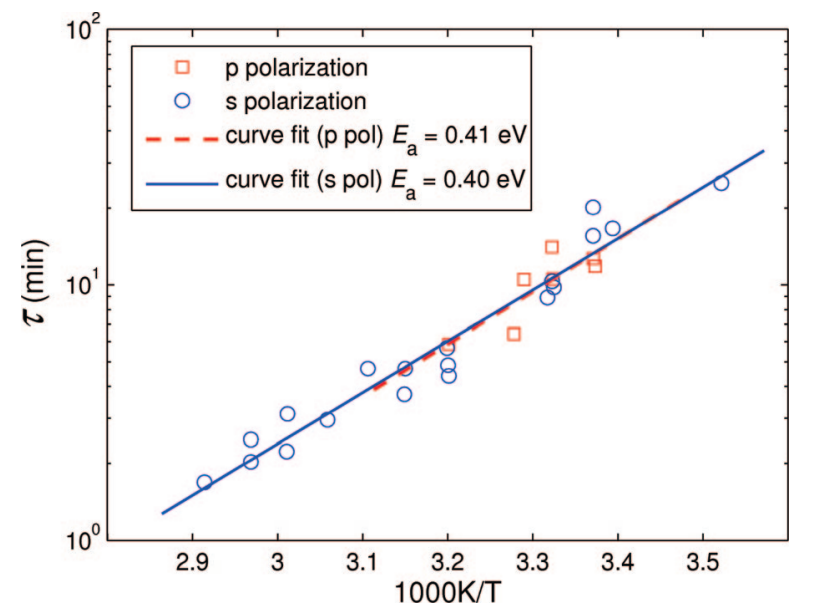

Figure 7. Arrhenius plot of time constants from the ionomer surface dynamics following the introduction of water at temperatures ranging from 10 to $70{ }^{\circ} \mathrm{C}$.

enrichment behavior of the PC ionomer with an added hydrophilic environment. It is evident that the movement of the PC endgroups from the aggregates in the bulk to the surface would entail cooperative interactions within the ionomer. Previous studies that used SHG to study polymer dynamics have found KWW behavior, but these were investigations of the bulk orientational relaxation dynamics, where the inversion symmetry of the bulk was purposely broken by a DC poling field. ${ }^{27,34} \mathrm{On}$ the other hand, first-order kinetics have been observed in a SFG study of the surface modification of poly(dimethylsiloxane). ${ }^{35}$ The 2D constraints of the surface are obviously different from the $3 \mathrm{D}$ constraints of the bulk and may thus contribute to the difference in molecular dynamics. For example, it has been demonstrated in SHG studies of liquid interfaces that interfacial dynamics can differ markedly from the corresponding bulk dynamics. ${ }^{36,37}$

Figure 7 displays the Arrhenius plot of the time constants from the ionomer surface dynamics following the introduction of water. The activation energy, $E_{\mathrm{a}}$, can be extracted from a curve fit to the equation:

$$
\tau=A \exp \left(\frac{E_{\mathrm{a}}}{k_{\mathrm{B}} T}\right)
$$

where $A$ is a constant, $k_{\mathrm{B}}$ is the Boltzmann constant, and $\tau$ is the time constant for the molecular dynamic process at temperature $T$. Data series are plotted for both s and p polarizations of the incident laser beam. It was found that the SHG signal levels were somewhat higher with the s polarization, which indicates that the ionomer surface has a larger $\chi^{(2)}$ in the $\mathrm{s}$ polarization direction. This would occur if, for example, the average polarization vector of the PC endgroups were more horizontal than vertical with respect to the ionomer surface. The 


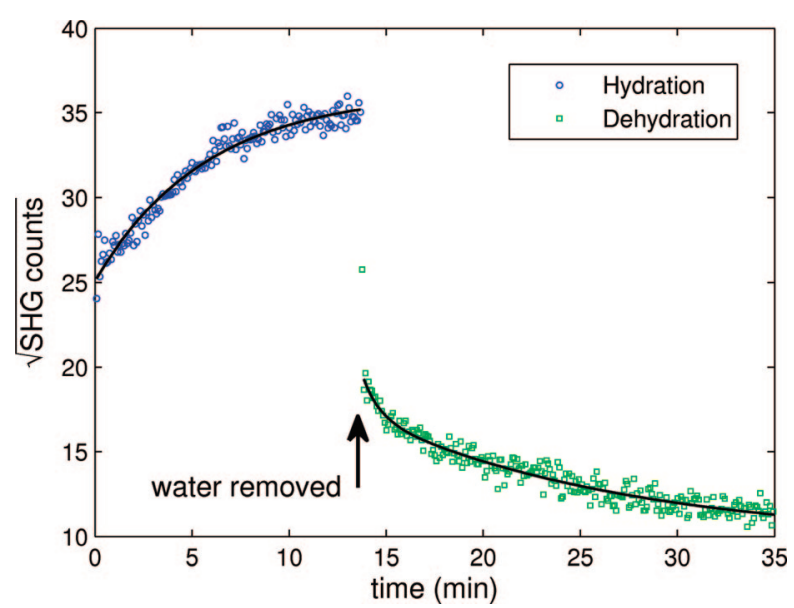

Figure 8. Hydration/dehydration cycle of PC ionomer carried out at $39.2{ }^{\circ} \mathrm{C}$. The time constant of the hydration cycle was $5.72 \mathrm{~min}$, while the dehydration cycle indicates two dynamic processes with time constants of $52 \mathrm{~s}$ and $13.5 \mathrm{~min}$ (corresponding curve fits indicated by solid black lines).

interaction of the ionomer surface with water is expected to induce a preferential orientation of these polar endgroups, which would also contribute to an increase in the SHG signal in addition to that due to the surface enrichment of endgroups from the bulk. However, it is not possible to provide more quantitative information about orientation without a closer study of the surface while controlling the polarization of both the incident and the nonlinearly scattered laser beams. ${ }^{38}$

The activation energies of the ionomer surface dynamics for the $\mathrm{s}$ polarization and $\mathrm{p}$ polarization directions were $0.40 \mathrm{eV}$ (95\% confidence bounds $=0.35-0.45 \mathrm{eV})$ and $0.41 \mathrm{eV}(95 \%$ confidence bounds $=0.10-0.71 \mathrm{eV})$, respectively. The similar activation energies of the $\mathrm{s}$ and $\mathrm{p}$ polarization measurements indicate that they correspond to the same dynamic process, which we attribute to the movement of the PC endgroups. Large changes in the ionomer surface energy after the introduction of water, as shown by contact angle measurements, ${ }^{29}$ also suggest the surface enrichment of PC endgroups from within the bulk. This migration of PC endgroups necessarily entails movement of the ionomer backbone. While SFG studies have shown that water-induced surface restructuring occurs immediately for side chain reorientations, ${ }^{18,39}$ it has also been demonstrated that surface restructuring involving movements of the polymer backbone can take place on much longer time scales. ${ }^{15}$

Backbone mobility depends on the free volume of the system and therefore is related to the glass transition temperature $T_{\mathrm{g}}{ }^{32}$ Consequently, the temperature dependence of such movements close to $T_{\mathrm{g}}$ normally follows Vogel-Fulcher-Tamann $(\mathrm{VFT})^{40-42}$ instead of the Arrhenius behavior observed here. This difference could be attributed again to differences in surface dynamics versus bulk dynamics because the free volume at the surface is presumably larger than that in the bulk. Indeed, some studies of polymer surfaces indicate a lower $T_{\mathrm{g}}$ of the polymer surface than that of the bulk. ${ }^{43,44}$

Dehydration Dynamics of the PC Ionomer. The dehydration dynamics of the ionomer surface are also worth investigation as they may not necessarily mirror the hydration dynamics. Figure 8 shows a hydration cycle followed by a dehydration cycle, carried out at $39.2^{\circ} \mathrm{C}$, in which the water was removed from the sample chamber and replaced with a nitrogen gas flow to aid in water evaporation from the ionomer surface. Although the cycles are qualitatively similar, we can observe significant differences. While the hydration dynamics reflect a process having a single time constant, two processes appear to govern the dehydration dynamics. The time constant for the hydration cycle, derived from a fit of eq 4 to the hydration data, was found to be $5.72 \mathrm{~min}$. The two time constants for the dehydration dynamics were found to be $52 \mathrm{~s}$ and $13.5 \mathrm{~min}$. During dehydration, the faster initial rate of change in the surface $\chi^{(2)}$ may be partially due to the evaporation of water molecules that are bound to the polar PC endgroups at the surface. The slower rate may reflect the migration of the $\mathrm{PC}$ endgroups back into the bulk. This larger time constant as compared to the hydration time constant would thus reflect a lesser driving force for the surface restructuring. A difference in the hydration/dehydration restructuring times was also observed in a SFG study of BioSpan grafted with poly(dimethylsiloxane) endgroups. ${ }^{15}$ However, in this case, the dehydration dynamics were faster than the hydration dynamics.

\section{Conclusion}

The environmental-induced surface dynamics of the biomimetic PC-functionalized PTMC ionomer has been studied and compared to its unfunctionalized counterpart using in situ second harmonic generation measurements. Whereas the nonpolar liquid $n$-hexane did not induce any surface dynamic processes in the ionomer under study, the presence of water initiated a Debyetype dynamic reaction at the surface of the PC ionomer, which had no equivalent in the unfunctionalized material. This firstorder reaction was attributed to a surface enrichment process of the functionalized ionomer in the hydrophilic environment involving movement of the PC endgroups from aggregates in the bulk to the surface. The time constant of the process was found to be about $6 \mathrm{~min}$, and the corresponding activation energy was $0.4 \mathrm{eV}$. The dehydration process of the PC-functionalized ionomer in nitrogen gas atmosphere could be described by two time constants, one slightly below $1 \mathrm{~min}$ and the other one just above $13 \mathrm{~min}$. The larger time constant in the dehydration dynamics, most likely corresponding to migration of the PC endgroups back into the bulk, reflects a lesser driving force in terms of PC concentration gradient as compared to the opposite process during hydration.

The results presented in this work demonstrate that SHG measurements are well suited for the study of polymer surface restructuring dynamics in response to environmental changes. Such information is invaluable for the successful design and implementation of biomimetic polymers intended for biomedical applications as it is the interface between the biomimetic polymer and the body that plays a major role in functional properties like hemocompatibility and drug delivery characteristics.

Acknowledgment. We are grateful to Dr. Fredrik Nederberg for the synthesis of the polymer samples. The Knut and Alice Wallenberg Foundation, The Swedish Research Council, as well as the Swedish Foundation for Strategic Research are greatly acknowledged for financially supporting our work.

\section{References and Notes}

(1) Andrade, J. D. Polymer Surface Dynamics; Plenum: New York, 1988.

(2) Lee, S. H.; Ruckenstein, E. J. Colloid Interface Sci. 1987, 120, 529-536.

(3) Hayward, J. A.; Chapman, D. Biomaterials 1984, 5, 135-142.

(4) Zalipsky, S. Bioconjugate Chem. 1995, 6, 150-165.

(5) Ruckenstein, E.; Gourisankar, S. V. J. Colloid Interface Sci. 1985, 107, 488-502.

(6) Williams, C. T.; Beattie, D. A. Surf. Sci. 2002, 500, 545-576.

(7) Shen, Y. R. Nature 1989, 337, 519-525.

(8) Shen, Y. R. Surf. Sci. 1994, 299, 551-562. 
(9) Chen, Z. Polym. Int. 2007, 56, 577-587.

(10) Marrucci, L.; Paparo, D.; Cerrone, G.; de Lisio, C.; Santamato, E.; Solimeno, S.; Ardizzone, S.; Quagliotto, P. Opt. Laser Eng. 2002, 37, 601610 . 2003

(11) Shen, Y. R. The Principles of Nonlinear Optics; Wiley: New York,

(12) Guyot-Sionnest, P.; Hunt, J. H.; Shen, Y. R. Phys. Rev. Lett. 1987, 59, 1597-1600.

(13) Zhuang, X.; Miranda, P. B.; Kim, D.; Shen, Y. R. Phys. Rev. B 1999, 59, 12632-12640.

(14) Dreesen, L.; Humbert, C.; Hollander, P.; Mani, A. A.; Ataka, K.; Thiry, P. A.; Peremans, A. Chem. Phys. Lett. 2001, 333, 327-331.

(15) Zhang, D.; Ward, R. S.; Shen, Y. R.; Somorjai, G. A. J. Phys. Chem. B 1997, 101, 9060-9064.

(16) Hopkins, A. J.; McFearin, C. L.; Richmond, G. L. Curr. Opin. Solid State Mater. 2005, 9, 19-27.

(17) Chen, C. Y.; Clarke, M. L.; Wang, J.; Chen, Z. Phys. Chem. Chem. Phys. 2005, 7, 2357-2363.

(18) Wang, J.; Paszti, Z.; Even, M. A.; Chen, Z. J. Am. Chem. Soc. 2002, 124, 7016-7023.

(19) Dannenberger, O.; Buck, M.; Grunze, M. J. Phys. Chem. B 1999, 103, 2202-2213.

(20) Salafsky, J. S.; Eisenthal, K. B. J. Phys. Chem. B 2000, 104, 77527755

(21) Salafsky, J. S.; Eisenthal, K. B. Chem. Phys. Lett. 2000, 319, 435439

(22) Nederberg, F.; Bowden, T.; Nilsson, B.; Hong, J.; Hilborn, J. J. Am Chem. Soc. 2004, 126, 15350-15351.

(23) Nederberg, F.; Bowden, T.; Hilborn, J. Macromolecules 2004, 37, 954-965.

(24) Nederberg, F.; Watanabe, J.; Ishihara, K.; Hilborn, J.; Bowden, T. Biomacromolecules 2005, 6, 3088-3094.

(25) Welch, K.; Nederberg, F.; Bowden, T.; Hilborn, J.; Strømme, M. Langmuir 2007, 23, 10209-10215.
(26) Apostoluk, A.; Nunzi, J. M.; Lee, K. W. Opt. Commun. 2006, 263, $337-341$.

(27) Cecchetto, E.; Moroni, D.; Jerome, B. J. Phys.: Condens. Matter 2005, 17, 2825-2842.

(28) Demtröder, W. Laser Spectroscopy: Basic Concepts and Instrumentation; Springer: Berlin, 2003.

(29) Nederberg, F.; Bowden, T.; Hilborn, J. Polym. Adv. Technol. 2005, 16, 108-112.

(30) Prasad, P. N.; Williams, D. J. Introduction to Nonlinear Optical Effects in Molecules and Polymers; Wiley: New York, 1991.

(31) Debye, P. Polar Molecules; Chemical Catalogue Co.: New York, $1929 ;$ p 94

(32) Schönhals, A. In Broadband Dielectric Spectroscopy; Kremer, F.,

Schönhals, A., Eds.; Springer-Verlag: Berlin, 2003; pp 225-293.

(33) Williams, G. J. Non-Cryst. Solids 1991, 131, 1-12.

(34) Hall, D. B.; Hamilton, K. E.; Miller, R. D.; Torkelson, J. M. Macromolecules 1999, 32, 8052-8058.

(35) Ye, H.; Gu, Z.; Gracias, D. H. Langmuir 2006, 22, 1863-1868.

(36) Antoine, R.; Tamburello-Luca, A. A.; Hebert, P.; Brevet, P. F.;

Girault, H. H. Chem. Phys. Lett. 1998, 288, 138-146.

(37) Zimdars, D.; Dadap, J. I.; Eisenthal, K. B.; Heinz, T. F. J. Phys. Chem. B 1999, 103, 3425-3433.

(38) Zhang, W. K.; Wang, H. F.; Zheng, D. S. Phys. Chem. Chem. Phys. 2006, 8, 4041-4052

(39) Wang, J.; Woodcock, S. E.; Buck, S. M.; Chen, C. Y.; Chen, Z. J. Am. Chem. Soc. 2001, 123, 9470-9471.

(40) Vogel, H. Phys. Z. 1921, 22, 645.

(41) Tammann, G.; Hesse, W. Z. Anorg. Allg. Chem. 1926, 156, 245.

(42) Fulcher, G. S. J. Am. Chem. Soc. 1925, 8, 339

(43) Kajiyama, T.; Tanaka, K.; Takahara, A. Polymer 1998, 39, 46654673 .

(44) Hammerschmidt, J. A.; Gladfelter, W. L.; Haugstad, G. Macromolecules 1999, 32, 3360-3367.

JP804070K 